\title{
Exploring the Activity and Specificity of Gold Nanoparticle-Bound Trypsin by Capillary Electrophoresis with Laser-Induced Fluorescence Detection
}

\author{
Yu-Fen Huang, Chih-Ching Huang, and Huan-Tsung Chang* \\ Department of Chemistry, National Taiwan University, Taipe, Taiwan, Republic of China
}

Received March 29, 2003. In Final Form: J une 15, 2003

\begin{abstract}
This paper describes the use of micellar electrokinetic chromatography (MEKC) and capillary zone electrophoresis (CZE) in conjunction with laser-induced fluorescence (LIF) detection for investigating the specificity of biocatalysis by trypsin when it is conjugated to gold nanoparticles (GNPs). I n the presence of sodium dodecyl sulfate (SDS), adsorption of the tryptic fragments on GNP-trypsin and on the capillary wall is reduced. As a result, the sensitivity and resolution of electropherograms of the tryptic fragments from bovine serum al bumin (BSA) is improved. MEKC-LIF measurements show clearly that thespecificity of GNP-trypsin differs from that of free trypsin and that the tryptic digest of GNP-BSA is significantly different from theGNP-trypticdigest of BSA. Wehaveused CZE - LIF toobservedifferences in the biocatalytic activity of trypsin and GN P-trypsin. Changes in theel ectropherograms provideinformation of the progress of digestion and indicate that the activity of GNP-trypsin is lower than that of free trypsin. The results of this study suggest that changes in the conformations and steric effects contribute to the loss of activity and changes in specificity of trypsin adsorbed on GNPs.
\end{abstract}

\section{Introduction}

Advances in nanotechnology allow the synthesis and fabrication of numerous novel nanoparticles (NPs) for different purposes, such as in el ectronics, as sensors, and as catalysts, because of the uniqueelectronic, optical, and catalytic properties that result from their size. ${ }^{1-3}$ These properties arisefor thefollowing reason: For a given mass of gold, thesurfacearea increases with decreasing particle size. In addition, the selectivity and specificity of NPs toward substrates can be improved by controlling their sizes and shapes precisely and/or by modifying their surfaces.

Functional gold nanoparticles (GNPs) that are conjugated with biomolecules such as DNA, enzymes, and anti bodies have been made and demonstrated in a variety of fields, including biosensors, separation science, and catalysis. ${ }^{4-11}$ Recently, GNPs conjugated with enzymes, such as pepsin and fungal protease, have been synthesized and tested, with results showing that biocatalyticactivity and stability in aqueous solution is retained. ${ }^{10,11}$ The conjugation is believed to occur mainly through interac-

* Corresponding author: Tel/fax 011-886-2-2362-1963; e-mail changht@intu.edu.tw.

(1) Shipway, A. N.; Lahav, M.; Willner, I . Adv. Mater. 2000, 12, 993998.

(2) Vossmeyer, T.; Guse, B.; Besnard, I.; Bauer, R. E.; Müllen, K.; Yasuda, A. Adv. Mater. 2002, 14, 238-242.

(3) Shiraishi, Y.; Toshima, N. Colloids Surf. A 2000, 169, 59-66.

(4) Storhoff, J .J .; Elghanian, R.; Mucic, R. C.; Mirkin, C. A.; Letsinger,

R. L. J . Am. Chem. Soc. 1998, 120, 1959-1964.

(5) Maxwell, D. J .; Taylor, J . R.; Nie, S. J . Am. Chem. Soc. 2002, 124, 9606-9612.

(6) Wang, J .; Xu, D.; Kawde, A.-N.; Polsky, R. Anal. Chem. 2001, 73, $5576-5581$.

(7) Thanh, N. T. K.; Rosenzweig, Z. Anal. Chem. 2002, 74, 16241628.

(8) Zhang, C.; Zhang, Z.; Yu, B., Shi, J .; Zhang, X. Anal. Chem. 2002, 74, 96-99.

(9) Zanchet, D.; Micheel, C. M.; Parak, W.J .; Gerion, D.; Alivisatos, A. P. Nano Lett. 2001, 1, 32-35.

(10) Gole, A.; Dash, C.; Ramakrishnan, V.; Sainkar, S. R.; Mandale, A. B.; Rao, M.; Sastry, M. Langmuir 2001, 17, 1674-1679.

(11) Gole, A. Dash, C.; Soman, C.; Sainkar, S. R.; Rao, M.; Sastry, M. Bioconjugate Chem. 2001, 12, 684-690. tions of the gold surface with thiol groups of cysteine residues and amino groups of lysine residues of the enzymes; thestability of bioconjugated GNPsis attributed to steric and Coulombic repulsions. To retain their biocatalytic activities, the three-dimensional structures of enzymes must still exist when they are bound to GNPs. This situation can be achieved by carefully modifying the GNP surface with suitablecapping agents, such as citrate, and/or with spacers, such as poly(ethylene glycol). ${ }^{12}$ I n addition, thepH and ionicstrength of theaqueous solution are important factors that affect catalytic behavior. For example, the biological activity (selectivity and digestion efficiency) of pepsin-bi oconjugated GN Ps remains al most the same as it is in the bulk solution at pH 3.0 when glycine is used as the capping agent. ${ }^{10}$

Numerous spectroscopic tools, such as UV-vis absorption, fluorescence, Raman scattering, and I R spectroscopy, are commonly used to explore the stability and activity of bioconjugated GNPs. For example, the shifts in the so-called amide I and II bands in IR spectra of enzymes, and the $\pi-\pi^{*}$ band in fluorescence spectra that is due to thetryptophan residues, have been utilized to investigate adsorption, while acid-sol uble products (tryptophan and tyrosine residues) have been used to determine the biocatalytic ability of bound enzymes by measuring the UV absorbance of aromatic units at $280 \mathrm{~nm} .^{10,11,13}$ These techniques, however, do not provide direct information on the digestion products of NP-bound enzymes, which is information that is essential when investigating the biocatalytic activity and specificity of enzymes.

Capillary electrophoresis (CE) is a fast and efficient separation technique that can be used for the analysis of tryptic digests. ${ }^{14}$ It is a long-standing interest in our research group to study protein separation by CE in conjunction with laser-induced fluorescence (LIF) detec6678

12) Veiseh, M.; Zareie, M. H.; Zhang, M. Langmuir 2002, 18, 6671 -

(13) Keating, C. D.; Kovaleski, K. M.; Natan, M. J . J . Phys. Chem. B 1998, 102, 9404-9413.

(14) Welder, F.; McCorquodale, E. M.; Colyer, C. L. Electrophoresis 2002, 23, 1585-1590. 
tion. Over the past few years, we have developed techniques for the analysis of proteins subjected to electroosmotic flow (EOF) by using an Nd:Y AG laser with its output at $266 \mathrm{~nm} .{ }^{15,16}$ Although thesemethods allow the analysis of traces of proteins containingtryptophan, tyrosine, and/ or phenylalanine residues, fluorescence that is quenched by SDS and acids remains problematic.

Despite its ability to analyze traces of proteins from complicated samples, such as urine and cerebrospinal fluids, CE-LIF has not been tested for the analysis of bioconjugated NPs. In the case of using GNPs, the two main problems arefluorescencequenching and adsorption of GNPs on the capillary wall. The native fluorescence of proteins is quenched because of energy transfer between proteins (or peptides) with maximum excitation wavelengths at ca. $340 \mathrm{~nm}$ and GNPs with high absorption coefficients in theUV spectral range. Adsorption of GNPs on thecapillary wall causes a change in theelectroosmotic flow (EOF), which leads to irreproducibility of results. Loss of resolution is another problem if the peptides are adsor bed strongly onto GN Ps because this process affects their electrophoretic mobilities.

Theprimary goal of this work was to develop techniques for exploring thespecificity and bioactivity of GNP-bound trypsin (GNP-trypsin). We have analyzed the tryptic digests of bovine serum albumin (BSA) by micellar electrokineticchromatography (MEKC) - LIF usingan Nd: YAG laser at $266 \mathrm{~nm}$ and explored the effect of the SDS concentration and $\mathrm{pH}$ on the resolution and sensitivity. Theel ectropherograms reveal the different bioactivity and specificity of GNP-trypsin and trypsin. To provide kinetic information, we have also analyzed the tryptic digests by capillary zone electrophoresis (CZE) - LIF using a BSAspecific dye, al bumin blue 580 (AB 580).

\section{Experimental Section}

Materials. SDS, proteins, and all chemicals for preparing buffers were obtained from Sigma (St Louis, MO). AB 580 was purchased from M ol ecular Probes (Eugene, OR). TB buffers were prepared fromtris(hydroxymethyl)aminomethane(Tris) adjusted with boric acid to values of $\mathrm{pH}$ ranging from 8.0 to 10.0. In this paper, the molarity of TB buffer refers to that of Tris. A $10 \mathrm{mM}$ stock solution of AB 580 was prepared in methanol. Solutions of BSA and trypsin were prepared in water and stored at $4{ }^{\circ} \mathrm{C}$.

Capillary Electrophoresis Apparatus. The basic design of the separation system has been reported previously ${ }^{17}$ and is described herebriefly. A high-vol tage power supply from Gamma High Voltage Research (Ormond Beach, FL) was used to drive electrophoresis. The entire system was enclosed in a black box with a high-voltage interlock. The high-voltage end of the separation system was housed in a plexiglass box for safety. An EPO-5000 pulsed Nd:YAG laser at $266 \mathrm{~nm}, 3000 \mathrm{~Hz}$ output, from Continuum (Santa Clara, CA) was used to induce intrinsic fluorescence. When AB 580 was used for kinetic studies, a 1.5mW He-Nelaser with 594 nm output from Melles Griot (I rvine, CA) was used for excitation and a 620-nm interferencefilter was used to block the scattered light. I n both cases, the emitted light was collected with a $10 \times$ objective (numerical aperture $=0.25$ ) before it reached a Hamamatsu R928 photomultiplier tube (Shizuoka-Ken, J apan). The amplified current was transferred directly through a 10-k $\Omega$ resistor toa B orwin 24-bit A/D interface at $5 \mathrm{~Hz}$ (J MBS Developments, LeF ontanil, France) and the data werestored in a personal computer. F used-silica capillaries from Polymicro Technologies (Phoenix, AZ) with $75 \mu \mathrm{m}$ i.d. and 365 $\mu \mathrm{m}$ o.d. were used for protein separations without any coating process.

(15) Tseng, W.-L.; Chang, H.-T. Anal. Chem. 2000, 72, 4805-4811. (16) Tseng, W.-L.; Lin, Y.-W.; Chang, H.-T. Anal. Chem. 2002, 74, 4828-4834.

(17) Chen, H.-S.; Chang, H.-T. Anal. Chem. 1999, 71, 2033-2036.
Synthesis of GNPs. The preparation of 13-nm-diameter GNPs was achieved according to a reported method, described here briefly. ${ }^{18} \mathrm{An}$ aqueous solution of $\mathrm{HAuCl}_{4}(1 \mathrm{mM}, 250 \mathrm{~mL})$ was brought to a vigorous boil with stirring in a round-bottom flask fitted with a reflux condenser, and then trisodium citrate (38.8 mM , $25 \mathrm{~mL}$ ) was added rapidly. The solution was boiled for another $15 \mathrm{~min}$, during which time the color of the solution changed from pale yel low to deep red. The solution was cooled to room temperature with continued stirring.

Formation of GNP-Trypsin and GNP-BSA. The GNP solution prepared abovewas diluted by a factor of 3 with a glycine buffer $(75 \mathrm{mM}, \mathrm{pH}$ 9.0). Trypsin $(0.1 \mathrm{mM}, 0.5 \mathrm{~mL})$ or BSA $(0.1$ $\mathrm{mM}, 0.5 \mathrm{~mL}$ ) was added with stirring to a portion of the diluted solution containing the GNPs $(50 \mathrm{~mL} ; 50 \mathrm{mM}$ glycine buffer, $\mathrm{pH}$ 9.0). Thesolution was equilibrated for $1 \mathrm{~h}$ beforebeing centrifuged at $18000 \mathrm{rpm}$ for $20 \mathrm{~min}$ to remove the uncoordinated trypsin or BSA remaining in solution. The precipitate obtained was subjected to three repeated wash cycles involving rinsing with $50 \mathrm{mM}$ glycine buffer $(50 \mathrm{~mL})$ and centrifuging at $18000 \mathrm{rpm}$ for $20 \mathrm{~min}$. Finally, the GNP-trypsin or GNP-BSA was suspended in the glycine buffer $(12.5 \mathrm{~mL})$ for further experiments.

Tryptic Digestion and Peptide Separation by CE. The digestion of $5 \mu \mathrm{M}$ BSA was carried out with $0.25 \mu \mathrm{M}$ trypsin or GNP-trypsin (equilibrated with $0.25 \mu \mathrm{M}$ trypsin) in the glycine buffer at $37^{\circ} \mathrm{C}$ overnight. The digestion of GNP-BSA was carried out with $0.25 \mu \mathrm{M}$ trypsin in theglycinebuffer at $37^{\circ} \mathrm{C}$ overnight. The tryptic digests were injected hydrodynamically into an uncoated capillary filled with $100 \mathrm{mM}$ TB buffers containing 0-80 mM SDS, pH 8.0, at a height of $22.5 \mathrm{~cm}$ for $10 \mathrm{~s}$, and the separation was conducted at $15 \mathrm{kV}$. For the kinetic studies, the digestion of $5 \mu \mathrm{M}$ BSA was carried out with $0.25 \mu \mathrm{M}$ trypsin or GNP-trypsin in the glycine buffer at $37{ }^{\circ} \mathrm{C}$ for a period of time ranging from $1 \mathrm{~min}$ to overnight. The digests or supernatant of the tryptic digests were injected hydrodynamically into an uncoated capillary filled with $100 \mathrm{mM}$ TB buffer containing 1 $\mu \mathrm{M} \mathrm{AB} 580$ at a height of $22.5 \mathrm{~cm}$ for $10 \mathrm{~s}$, and the separations were preformed at $15 \mathrm{kV}$.

Spectroscopic Measurement. To investigate the effect of SDS on the fluorescence of tryptic and GNP-tryptic digests, we collected fluorescence spectra using a ascoF P-6200 spectrometer (Tokyo, J apan) with excitation at $280 \mathrm{~nm}$. To investigate the change in the surface plasmon resonance (SPR) of the GNPs, we took UV-vis spectra on a J asco spectrophotometer. To characterize the secondary structures of proteins in the GNP-trypsin bioconjugates, we collected FTIR spectra on a J asco Fourier transform infrared spectrometer. Thesamples were prepared by drop-drying BSA or GNP-BSA on a $\mathrm{KBr}$ cell.

\section{Results and Discussion}

Effect of Background E lectrolyte on Separation. The role that $\mathrm{pH}$ plays in the analyses of proteins and peptides by CE - LIF with UV lasers has been recognized for a number of years. ${ }^{19} \mathrm{I} \mathrm{n}$ view of theintrinsicfluorescence of proteins and peptides, conducting analyses at a $\mathrm{pH}$ of ca. 10.0 generally is preferred. The role of $\mathrm{pH}$ in determining resolution, however, is more complicated. On one hand, adsorption of proteins and peptides on the capillary wall is lessened at high $\mathrm{pH}$-when they are both negatively charged-because of Coulombic repulsion. On the other hand, it is easier to achieve high resolution in capillary zone electrophoresis (CZE) at values of $\mathrm{pH}$ close to the values of pl of proteins and peptides, but generally this situation requires a deactivated capillary. On the basis of these considerations, CZE - LIF was tested for the separation of tryptic digests of $\mathrm{BSA}$ at values of $\mathrm{pH}$ of 8.0, 9.0, and 10.0. A loss of resolution was observed at $\mathrm{pH} 10.0$, while peak broadening occurred at $\mathrm{pH} 8.0$ with only three peaks being detected. As shown in Figure 1A, the best result, in terms of resolution, was at $\mathrm{pH}$ 9.0. The number

(18) Grabar, K. C.; Freeman, R. G.; Hommer, M. B.; Natan, M. J Anal. Chem. 1995, 67, 735-743. 101.

(19) Tseng, W.-L.; Chang, H.-T. J . Chromatogr. A 2001, 924, 93- 


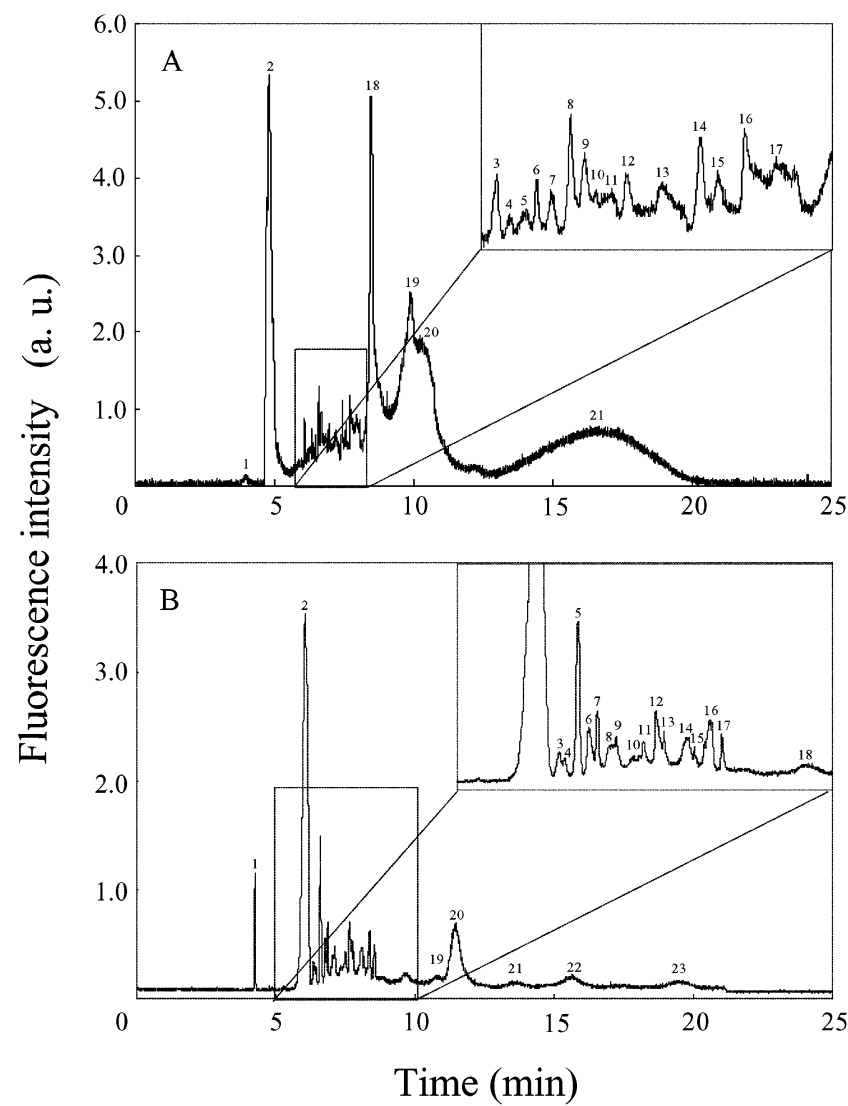

Figure 1. Capillary el ectropherograms displaying the separation of tryptic digests of BSA (A) in $100 \mathrm{mM}$ TB (pH 9.0) and (B) in $100 \mathrm{mM}$ TB (pH 8.0) containing $60 \mathrm{mM}$ SDS and detected with an Nd:YAG pulsed laser. Numbers denotedifferent tryptic fragments. Conditions: capillary total length $=40 \mathrm{~cm}$; effective length $=30 \mathrm{~cm}$; hydrodynamic injection height of $22.5 \mathrm{~cm}$ for $10 \mathrm{~s}$; separation voltage $15 \mathrm{kV}$. The peak numbers may not represent the same peptide fragments in the two electropherograms.

of peaks, however, was less than the total of 26 expected, indicating that further improvement in resolution was required. Two broad peak profiles at the migration times around 10 and $17 \mathrm{~min}$ indicate that adsorption of the fragments also took place.

Tofurther improveresolution, wetested the possibility of using MEKC-LIF, a techniquein which thebackground electrolytes contain SDS micelles. In the presence of these micelles, theseparation is preferred at $\mathrm{pH} 8.0$ rather than $\mathrm{pH}$ 9.0. This preference is mainly becausethepartitioning into SDS micelles of the tryptic fragments is greater at lower $\mathrm{pH}$ because of their lower negative-charge density. Several broad peaks and asymmetric peak profiles (not shown) indicated that adsorption is problematic at low SDS concentrations (<40 mM). With increasing SDS concentration, the peaks corresponding to the tryptic fragments became sharper and the migration times for some of the tryptic fragments became longer, thereby further improving theresolution. Theseparation was most successful at $60 \mathrm{mM}$ SDS, as is shown in Figure 1B. The resolution did not improve with further increases in SDS concentration, which instead caused instability of the baseline and irreproducibility of results, mainly because of problems associated with the fluorescent background (impurities from SDS) and J oule heating. It is important to note that SDS caused intrinsicfluorescence quenching of proteins such as BSA but not, or only slightly, of the tryptic fragments. The existence of this phenomenon is supported by the observation that the fluorescence
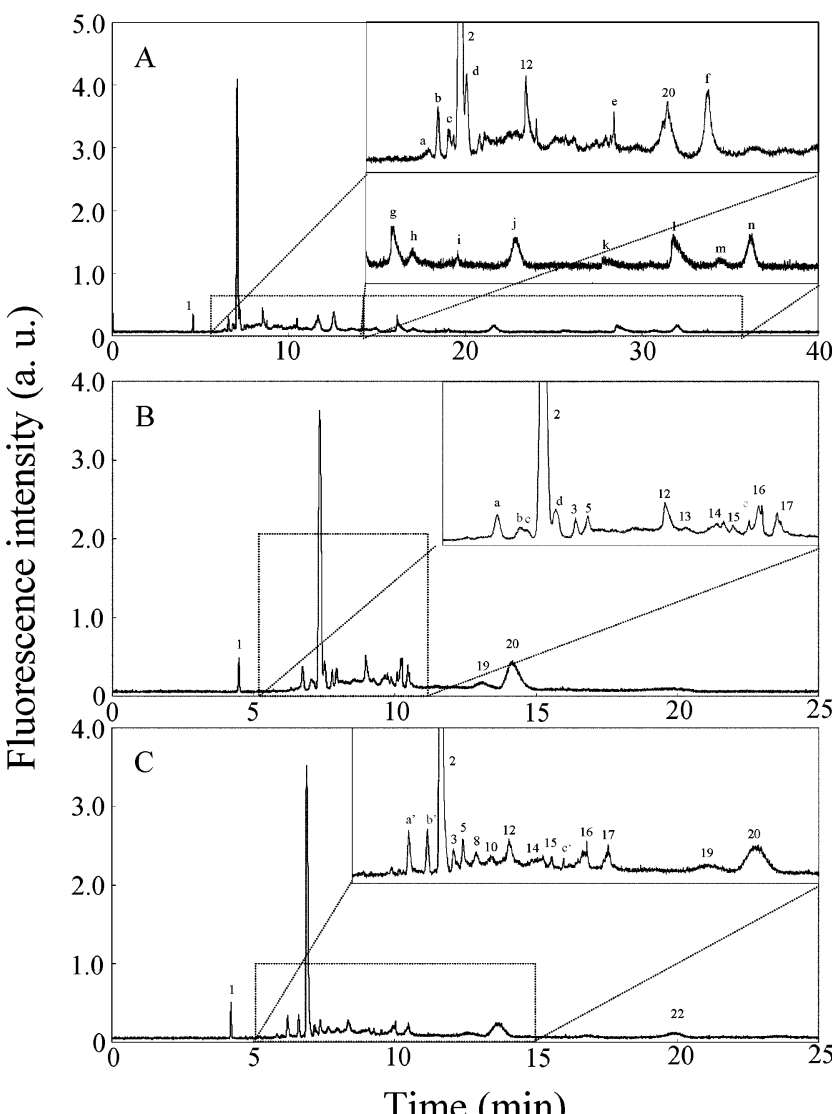

Figure 2. Electropherograms of (A) GNP-trypticdigest of BSA, (B) tryptic and GNP-tryptic digests of BSA, (C) GNP-tryptic digest of BSA after centrifugation. Letters denote the tryptic fragments obtained when only the GNP-trypsin was used. Conditions: separation buffer $100 \mathrm{mM}$ TB, pH 8.0, containing $60 \mathrm{mM}$ SDS. Other conditions are the same as those in Figure 1.

intensities of several resolved tryptic fragments (such as peaks 2, 5, and 12 in Figure 1B) remained al most constant at different SDS concentrations. This feature suggests that lack of interference is an advantage of conducting peptide analyses by this method, since proteins and enzymes are not detectable because of quenching by SDS.

Specificity of GNP-Trypsin and Trypsin. The developed MEKC-LIF method addressed above was applied to the analysis of GNP-tryptic digests of BSA. The electropherogram in Figure 2A displays several new peaks, marked $a-n$, but it lacks the peaks 3-11 and 1319 that were observed in Figure 1B. This result suggests that the specificity of GNP-trypsin is different from that of trypsin. A second explanation of this observation might be due to interactions of the GNPs with the capillary wall and with the tryptic fragments. To support these hypotheses, we conducted a tryptic digestion of BSA using a mixturecomposed of both freetrypsin and GNP-trypsin. The electropherogram shown in Figure 2B differs from those shown in either Figure 1B (trypsin alone) or Figure 2A (GNP-trypsin alone), with some peptide fragments appearing to be formed from digestion by freetrypsin and others by GN P-trypsin. To minimizethe effect that GNPs might haveon theresolution of theseparation, weinjected the sample after centrifugation, and the subsequent el ectropherogram is shown in Figure 2C. Theappearance of peaks 8 and 10 after centrifugation indicates that the corresponding tryptic fragments werelikely to have been desorbed from the GNP-trypsin by this process. On the other hand, peaks cand d disappeared after centrifugation, 

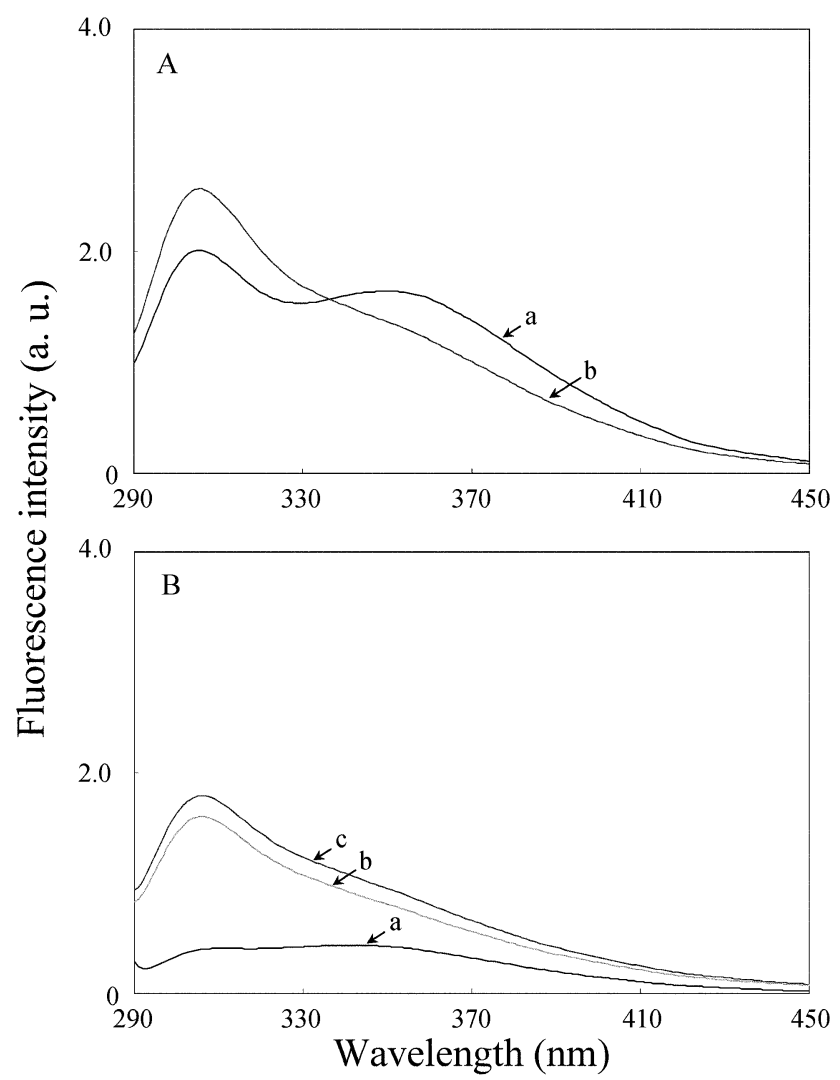

Figure 3. (A) Fluorescence spectra of the tryptic digests of BSA in the absence (curve a) and presence (curve b) of $60 \mathrm{mM}$ SDS. (B) Fluorescence spectra of the GNP-tryptic digests of BSA (curvea) and of the supernatant in the presence of $60 \mathrm{mM}$ SDS that was added after (curve b) and before (curve c) centrifugation.

indicating that thesetryptic fragments might havestrong interactions with the GNP-trypsin. Apparently, the different electropherogram patterns shown in Figures $1 \mathrm{~B}$ and $2 A, C$ indicate that the specificity of GNP-trypsin is different from that of trypsin, presumably because of the different conformations of theenzymes when immobilized.

To investigate the impact of SDS on the sensitivity of detection by fluorescence, we added SDS to the tryptic digest. Figure 3A show that two bands (in curve a) with wavel ength maxima at 308 and $350 \mathrm{~nm}$ in the emission spectrum of the tryptic digest are transformed into one band with a shoulder (in curve b) in the presence of 60 $\mathrm{mM}$ SDS. The results reveal that interactions take place to some extent between the tryptic fragments and SDS micelles. Theincreasein thefluorescenceintensity around $308 \mathrm{~nm}$ indicates the advantage of analyzing peptides by MEKC-LIF. Note that the maximum fluorescence wavelength of BSA has been shifted from 338 to $310 \mathrm{~nm}$ and that the intensity has decreased by a factor of 1.4 under these conditions. To show that fluorescence quenching is caused by the GNPs and that SDS plays a rolein desorbing the tryptic fragments from the GNPs, we collected the fluorescence spectra of the GNP-tryptic digests of BSA in theabsenceand presence of $60 \mathrm{mM}$ SDS. Figure $3 B$ shows that the fluorescence intensity of the tryptic fragments around $308 \mathrm{~nm}$ is very weak (curvea), which supports our belief that energy transfer occurs between GNPs and the trypticfragments. This result also confirms that thetryptic fragments are adsorbed on the surface of GNP-trypsin in the absence of SDS. The fluorescence intensity of the supernatant at ca. $308 \mathrm{~nm}$ increases dramatically (curve b) in the presence of SDS, which suggests that the tryptic fragments are easily separated from GNP-trypsin by
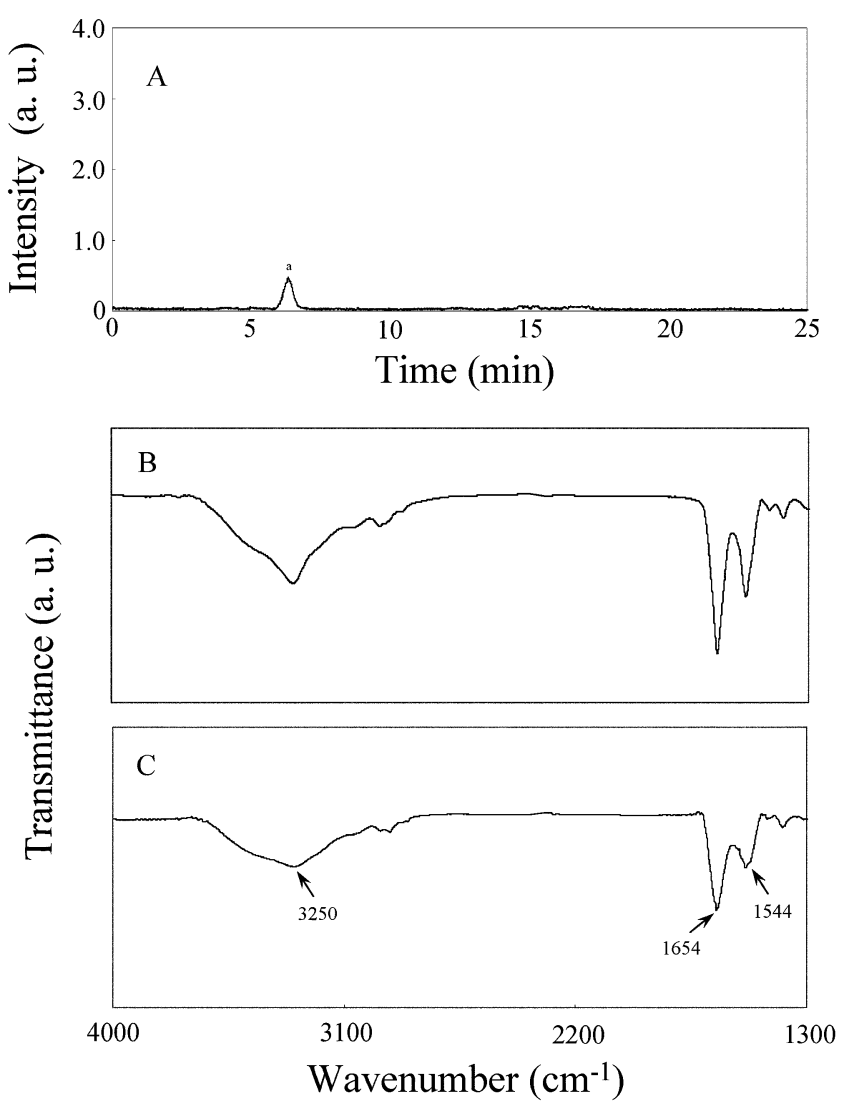

Figure 4. (A) Electropherogram of tryptic digests of GNP$B S A$. The peak is due to a tryptic fragment. Conditions are the sameas thosein Figure 2. (B) FTIR spectrum of BSA. (C) FTIR spectrum of GNP-BSA.

simple centrifugation in the presence of SDS. This observation suggests that some of the peptides adsorb physically (weakly) on the surface of the GNP-trypsin. By comparison, thefluorescenceincreased (curvec) when SDS was added to the digest prior to centrifugation, which indicates that someof the peptides might adsorb strongly. This result suggests that adding SDS is an effective method for therecovery of peptides bound to GNP-trypsin and supports our reasoning discussed above (Figure 2).

Next we investigated the digestion of GNP-BSA with trypsin under thesame conditions. Figure 4A displays an el ectropherogram with only one peak, which is a situation much different from the results shown in Figures 1B and 2. It is important to notethat thepeak does not correspond to either GNP-BSA or to BSA. Similar results were found also in the cases of GNP-carbonic anhydrase, GNP- $\beta$ casein, and other systems. Aggregation of the GNPs is not a reason for this result, which is confirmed by the observation that no change occurred in the SPR band of the GNPs around $519 \mathrm{~nm}$. I ndeed, we have found that the GNPs are stable over a broad range of $\mathrm{pH}$ values (6.0$10.0)$ and at higher ionic strengths (>100 $\mathrm{mM})$ in the presence of BSA. The one peak detected suggests that most of the active sites are not available for trypsin once BSA is adsorbed on theGNPs. This hypothesis is supported by the fact that BSA contains 35 cysteine residues and 60 Iysineresidues that are bel ieved to beabletoform covalent bonds to the GNPs. We conducted IR measurements to investigate the interactions between BSA and the GNPs. The feature at ca. $3250 \mathrm{~cm}^{-1}$ in theFTIR spectrum shown in Figure 4B arises from the $\mathrm{N}-\mathrm{H}$ stretching vibration mode in the BSA molecules. Figure $4 C$ shows that this band becomes broad in GNP-BSA, which indicates that binding occurs of the amino groups of BSA to the GNPS. 


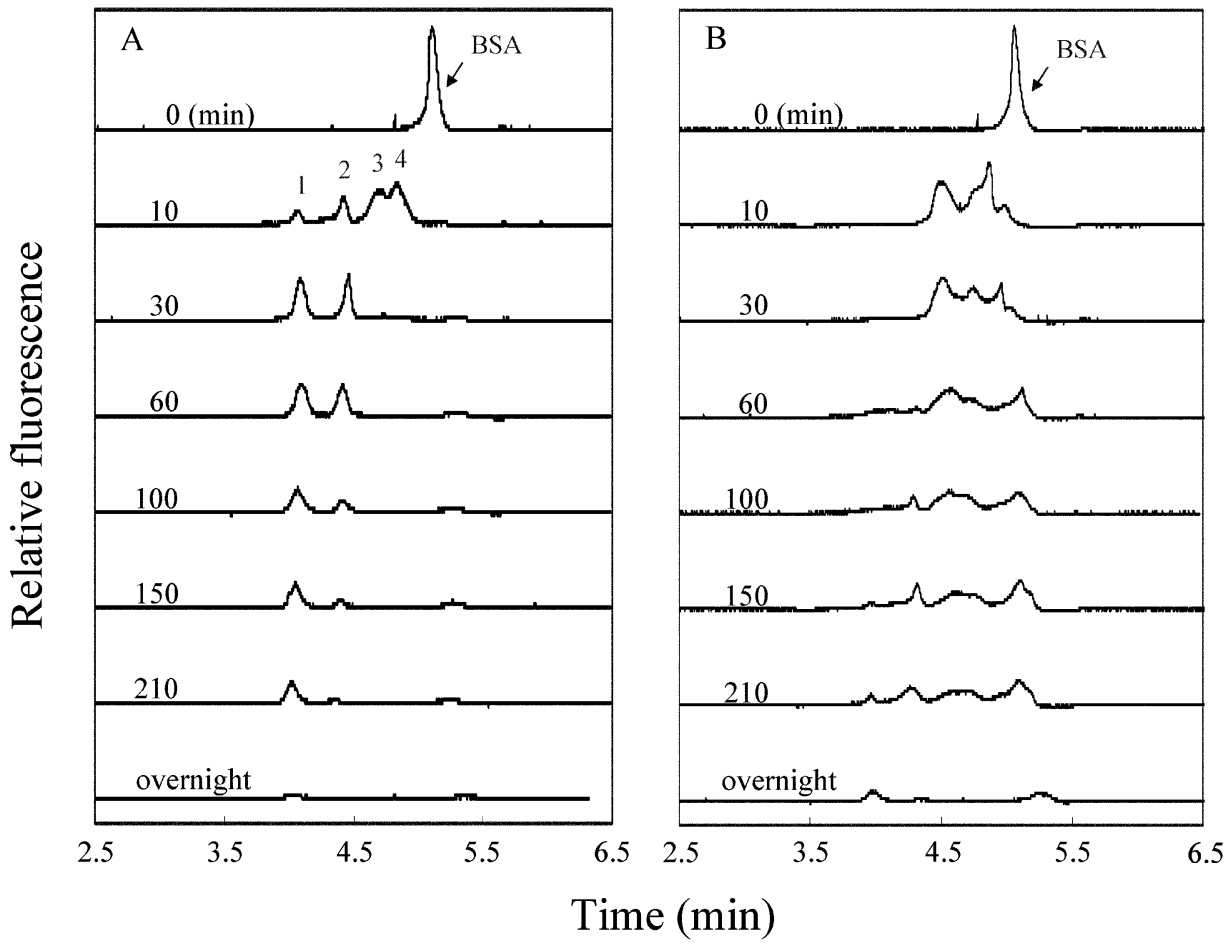

Figure 5. Electropherograms of (A) tryptic and (B) GNP-tryptic digests of BSA obtained by CZE -LIF with an He-Ne laser. The capillary was filled with $100 \mathrm{mM}$ TB (pH 8.5) containing $1 \mu \mathrm{M}$ AB 580. Other conditions are the same as those in Figure 1.

Thebands at ca. $1500-1700 \mathrm{~cm}^{-1}$ (amidebands) are broad, which shows that changes in the structure of BSA occur once it is adsorbed on the GNPs.

Kinetics. Because SDS and/or GN Ps cause quenching of the intrinsic fluorescence of BSA, the conditions used above are not suitable for kinetic studies. Thus, we devel oped a CZE - LIF technique that uses no SDS in the background electrolytes by using AB 580, a highly selective dye for BSA, to label BSA and an $\mathrm{He}-\mathrm{Nelaser}$ at $594 \mathrm{~nm}$ to excite the BSA-AB 580 complex. AB 580 alone is nonfluorescent, but it is strongly fluorescent onceit binds to BSA. ${ }^{20,21}$ Figure 5 shows the different peak profiles observed during the course of the reactions (times between 0 and $12 \mathrm{~h}$ ) when trypsin and GNP-trypsin were used to digest BSA. The peak corresponding to BSA disappeared within 10 min when trypsin was used, but some BSA remained when GNP-trypsin was used, a situation that confirms that the activity of trypsin is higher than that of GNP-trypsin. On the basis of changes in the peak height of BSA, we estimate that the digestion efficiency of free trypsin is about $39.8 \%$ in 1 min, which is much higher than that of GNP-trypsin (less than 1.0\%). The peak corresponding to BSA and peaks $1-4$ were still observed after digestion overnight with GNP-trypsin, which indicates clearly that its activity is lower than that of free trypsin. There is al so evidence that shows the differences in the progress of digestion. Four tryptic fragments and two (or three) GN P-tryptic fragments, respectively, bind to AB 580 after 10 min of digestion. Peak 1 appeared within

(20) Kessler, M. A.; Meinitzer, A.; Wolfbeis, O. S. Anal. Biochem. 1997, 248, 180-182.

(21) Tseng, W.-L.; Chiu, T.-C.; Weng, J .-M.; Chang, H.-T. J . Liq. Chromatogr. Relat. Technol. 2001, 24, 2971-2982.
10 min and its intensity increased with further reaction timeup to 60 min when trypsin was used, while it started to appear only after $60 \mathrm{~min}$ when GN P-trypsin was used. Theincrease in theintensity of peak 1 correlates with the decrease in the intensity of peaks 3 and 4 in the course of $30 \mathrm{~min}$, which indicates that the tryptic fragment corresponding to peak 1 is likely to be digested from fragments 3 and 4. Again, we suggest that the lower activity of GNP-trypsin relative to trypsin might be attributable to changes in protein conformation and/or steric effects.

\section{Conclusions}

Wehavedescribed an MEKC-LIF method for exploring, for the first time, the biocatalytic activity and specificity of bioconjugated GNPs. In this study, wehave found that adding SDS to the background el ectrolyte is extremely useful because the tryptic fragments desorb readily from the GNPs and fluoresce strongly in the presence of SDS micelles. The MEKC-LIF and CZE - LIF measurements show clearly that GNP-trypsin and trypsin possess different specificity, with the former having lower activity mainly because of changes in the enzyme's conformation. Although we used an inexpensive UV-pulsed laser in this study, the reported method can easily be applied to the investigation of the bioactivity and specificity of bioconjugated NPs using any commercial CE instruments with UV-vis light sources.

Acknowledgment. This work was supported by the National ScienceCouncil of Taiwan, the Republic of China (NSC 91-2113-M-002-052 and NSC 91-2120-M-002-001).

\section{LA0345410}

